\title{
TECNOLOGIAS NÃO FARMACOLÓGICAS E TEMPO DE TRABALHO DE PARTO E PARTO: REVISÃO SISTEMÁTICA SEM METANÁLISE
}

NON-PHARMACOLOGICAL TECHNOLOGIES AND LABOR AND DELIVERY TIME:

\author{
SYSTEMATIC REVIEW WITHOUT META-ANALYSIS
}

Kayo Henrique Jardel Feitosa Sousa ${ }^{1} *$ Aislan Ferreira Sena ${ }^{2} *$ Karla Joelma Bezerra Cunha $^{3}$

\section{RESUMO}

Objetivo: identificar, na literatura, associações entre o uso de tecnologias não farmacológicas e o tempo de trabalho de parto e parto. Métodos: revisão sistemática sem metanálise, incluindo somente ensaios clínicos randomizados. Fator de exposição: terapias não farmacológicas de alívio da dor. Desfecho: tempo de trabalho de parto e parto. Resultados: nove artigos foram identificados. Três não apresentaram eficácia relacionada ao tempo de trabalho de parto e parto, contudo, um obteve para os dois períodos do trabalho de parto e parto, dois para a duração total do trabalho de parto e parto, um para o período expulsivo de forma isolada e duração total do trabalho de parto e parto e dois somente para o período expulsivo. Conclusão: as tecnologias não farmacológicas de alívio da dor, aplicadas de forma isolada ou combinada, apresentam potencial efetivo no alívio da dor de parturientes e redução significativa do tempo de trabalho de parto e parto.

Palavras-chave: Trabalho de parto; Parto normal; Enfermagem Obstétrica; Terapias Complementares; Prática Clínica Baseada em Evidências.

\begin{abstract}
Objective: to identify, in the literature, associations between the use of non-pharmacological technologies and the time of labor and delivery. Methods: systematic review without meta-analysis, including only randomized controlled trials. Exposure factor: non-pharmacological pain relief therapies. Outcome: length of labor and delivery. Results: nine articles were identified. Three did not show efficacy related to the time of labor and delivery, however, one obtained for both periods of labor and delivery, two for the total duration of labor and delivery, one for the expulsion period in isolation and total duration of labor and delivery and two only for the expulsion period. Conclusion: non-pharmacological pain relief technologies, applied in isolation or in combination, have an effective potential in relieving the pain of parturients and significantly reducing labor and delivery time.
\end{abstract}

Keywords: Labor, Obstetric; Natural Childbirth; Obstetric Nursing; Complementary Therapies; Evidence-Based Practice.

\footnotetext{
${ }^{1}$ Enfermeiro Obstétrico. Universidade Federal do Rio de Janeiro (UFRJ), Escola de Enfermagem Anna Nery (EEAN). Rio de Janeiro, RJ, Brasil. E-mail: kayohenriquejardel@ hotmail.com ORCID: https://orcid.org/0000-0002-0901-7752. ${ }^{2}$ Enfermeiro Obstétrico. Hospital Santo Antônio. Blumenau, SC, Brasil. Email: aislansena@ hotmail.com ORCID: https://orcid.org/0000-0002-1994-5368.

${ }^{3}$ Enfermeira Obstétrica. Centro Universitário Santo Agostinho. Teresina, PI, Brasil. Email: karlaenfa@yahoo.com.br ORCID: https://orcid.org/0000-0002-2134-4844.
} 


\section{INTRODUÇÃO}

O parto e nascimento são considerados fenômenos naturais e por muito tempo configurou-se como evento no qual mulher era protagonista, sendo acompanhada por parteiras. Contudo, por volta do século XVII observou-se um processo de institucionalização do parto, com a inserção dos médico-cirurgiões no cenário assistencial, dando lugar às intervenções desnecessárias e sem critérios, aumentando o número de cesarianas e reduzindo a autonomia da mulher ${ }^{(1)}$.

No Brasil, vive-se atualmente um processo de transição do modelo de atenção ao parto e nascimento, com resgate do protagonismo da mulher e respeito à sua dignidade, buscando uma assistência humanizada. Destaca-se, nesta direção, o documento Tecnologias apropriadas para o parto e nascimento, lançado pela Organização Mundial da Saúde (OMS), em 1985, inserindo as boas práticas de atenção ao parto $\mathrm{e}$ nascimento no rol de atividades da obstetrícia visando redução da mortalidade maternoinfantil, meta de desenvolvimento do milênio $^{(2)}$.

Cabe ressaltar também, o Relatório Técnico Assistência ao Parto Normal: um guia prático $^{(3)}$ e as Diretrizes Nacionais de Assistência ao Parto Normal ${ }^{(4)}$, que propõem mudanças no cenário assistencial, com resgate do parto como fenômeno natural, utilização na prática clínica das melhores evidências científicas, acesso às tecnologias apropriadas e estímulo da atuação da enfermagem obstétrica.

Várias pesquisas visaram avaliar a utilização e eficácia das boas práticas de atenção ao parto e nascimento. Estudo de base hospitalar com 23.894 puérperas demonstrou redução no número de cesáreas e aumento de partos normais sem intervenções em gestantes de risco obstétrico habitual. Ainda, demonstrou que as boas práticas foram usadas em menos de $50 \%$ das mulheres em trabalho de parto (TP), a prevalência da manobra de Kristeller foi de $37 \%$, enquanto de posição litotômica de 92\%, 40\% fizeram uso de ocitocina e amniotomia e em $56 \%$ ocorreu episiotomia, revelando que as mulheres ainda estão expostas a riscos de iatrogenia ${ }^{(5)}$.

Outro estudo demonstrou que intervenções educativas com os profissionais envolvidos na assistência ao parto e nascimento são de suma importância neste processo de humanização do parto e nascimento, tendo em vista que, após intervenção houve aumento na taxa de parto normal, da presença de acompanhante durante o TP e do uso das posições verticalizadas, com significativa redução das taxas de amniotomia, posição litotômica e do uso de ocitocina intravenosa ${ }^{(6)}$. Outro fator 
contributivo para a mudança deste cenário é a inserção da enfermagem obstétrica, como recomenda a OMS. Estudos demonstraram melhorias na qualidade da assistência com o trabalho multiprofissional e integrado entre médicos e enfermeiros obstétricos ${ }^{(7-8)}$.

Apesar de todos os avanços tecnológicos e incremento da assistência com as boas práticas de atenção ao parto e nascimento, o TP e o parto normal continuam sendo assustadores para as mulheres, em especial, às primíparas, com representação social negativa em virtude de um conjunto de significados preconcebidos culturalmente aliados à dor, sofrimentos e angústias ${ }^{(9)}$.

O fenômeno "não sentir dor" é apontado como o principal motivo para a preferência das mulheres a cesariana, enquanto a "melhor recuperação no pós-parto" para a via de parto vaginal, pois, consideram a recuperação pósparto cesariana uma experiência mais demorada e dolorosa. Outros fatores merecem atenção quanto à preferência pela via de parto, como a história obstétrica e o tipo de parto anterior. Esta experiência anterior pode ser um motivador para a escolha do tipo de parto atual e deve ser considerada quando da elaboração do Plano de Parto ${ }^{(10)}$.

A dor é um fenômeno fisiológico circunscrito ao processo parturitivo e que neste contexto não está relacionada a doenças, desta forma, fornecer informações sobre o funcionamento do corpo, a fisiologia do parto e os mecanismos de dor podem ajudar as mulheres a ressignificar o sofrimento aliado ao TP. Neste sentido, recomenda-se que os profissionais de saúde considerem suas crenças e valores que podem estar influenciando sua maneira em lidar com a dor no TP e parto, visando garantir que sua assistência apoie a escolha da mulher, e ainda encorajar e divulgar amplamente as tecnologias não farmacológicas (TNF) de alívio da dor nas maternidades ${ }^{(4)}$.

As TNF são consideradas tecnologias leve-duras e devem ser encorajadas, podendo ser operacionalizados pelo acompanhante de escolha da mulher, contribuindo para o protagonismo da parturiente e promovendo conforto. Estudos de revisão integrativa demonstraram como TNF utilizadas no TP a(o): estimulação transcutânea, exercícios respiratórios, mudança de posição, deambulação, massagem, hidroterapia, crioterapia, assistência de doulas, aromoterapia, musicoterapia, acupuntura, acupressão e hipnose $\mathrm{e}^{(11-12)}$.

Dados consistentes apontam que o uso das TNF no TP e parto, provocam: diminuição da dor, do tempo do TP e parto, aumento da atividade uterina, oferecendo menor risco de sofrimento fetal e melhorando o conforto materno, redução dos índices de parto cesáreo e analgesia farmacológica, além de contribuir para a satisfação da mulher com 
o TP, favorecendo mudanças na representação social negativa do $\mathrm{TP}^{(1,13-15)}$.

Estudo bibliométrico apresentou o panorama mundial das pesquisas experimentais durante o período gestacional, revelando amplo processo de produção de conhecimento nesse âmbito, contudo, no cerne da temática proposta a esse estudo identificou-se uma exiguidade de estudos ${ }^{(16)}$. Revela-se, portanto, uma lacuna na pesquisa científica quanto à abordagem das TNF de alívio da dor durante o TP, com foco na duração do TP e parto. A busca por evidências científicas pode beneficiar os profissionais envolvidos na assistência obstétrica, especialmente trabalhadores da equipe de centros de parto normal e parturientes e a sua família visto o encorajamento ao protagonismo da mulher.

Esse estudo teve por objetivo: identificar, na literatura, associações entre o uso de tecnologias não farmacológicas e o tempo de trabalho de parto e parto.

\section{MÉTODOS}

Revisão sistemática da literatura sem metanálise, seguindo o fluxograma do Preferred Reporting Items for Systematic Reviews and Meta-Analyses (PRISMA) ${ }^{(17)}$.

O processo de elaboração desta revisão seguiu as etapas propostas nas Diretrizes Metodológicas para a Elaboração de Revisão Sistemática e Metanálise de Ensaios Clínicos
Randomizados, a saber: definição da questão de pesquisa estruturada no formato do acrônimo PICO, definição dos critérios de elegibilidade, justificativa para a revisão sistemática, busca dos potenciais estudos elegíveis, avaliação da elegibilidade dos estudos, extração de dados, apresentação, análise e aplicabilidade dos resultados ${ }^{(18)}$.

A questão de pesquisa foi definida por meio do acrônimo PICO (P: paciente, I: intervenção, $\mathrm{C}$ : controle e $\mathrm{O}$ : outcomes/desfecho). Considerou-se: P primíparas em trabalho de parto; I tecnologias não farmacológicas de alívio da dor; C - nenhuma intervenção ou qualquer outra; e O - redução do tempo de trabalho de parto, para a elaboração da seguinte pergunta: as tecnologias não farmacológicas de alívio da dor reduzem o tempo de trabalho de parto e parto em primíparas?

A busca foi realizada por dois pesquisadores de forma concomitante no período de julho de 2018. Os estudos foram identificados por meio de estratégias de buscas específicas em bases de dados com significativo impacto no contexto da saúde, quais sejam: Literatura Latino-Americana e do Caribe em Ciências da Saúde (LILACS), Cumulative Index of Nursing and Allied Health Literature (CINAHL), Scientific Electronic Library Online (SciELO), Web of Science (WOS), Scopus, Pubmed e Bases de Dados de Enfermagem (BDENF). 
Definiu-se para a busca dos artigos as seguintes frases booleanas, com o auxílio dos Descritores em Ciências da Saúde (DECS)/Medical Subject Heading (MESH): "dor" AND "trabalho de parto", "pain" AND "labor, obstetric", "trabalho de parto" AND "assistência ao parto" e "labor, obstetric" AND “midwifery".

Os critérios de elegibilidade foram: (1) ensaios clínicos randomizados (ECR) em texto completo, (2) estar publicado em português, inglês ou espanhol e (3) ter sido publicado no período de 2014 a 2018 (até a data da busca pelos pesquisadores), presenciase, mundialmente, nos últimos anos intenso debate sobre a humanização da assistência ao parto e nascimento, justificando assim o recorte temporal. Estudos foram excluídos devido aos seguintes motivos: (1) não abordava adequadamente a temática, (2) revisão, tese, dissertação, opinião de especialista, resumo, carta ao editor, editorial, estudo transversal/cross-sectional, estudo qualitativo, (3) ECR que não mostrou resultados para o outcomes/desfecho a ser analisado e (4) duplicados.

Após o levantamento dos dados, procedeu-se leitura minuciosa dos títulos e resumos para avaliação da elegibilidade, permitindo excluir significativa quantidade de relatos que não se adequavam a proposta do protocolo de pesquisa. Quando houve discordância, entre os pesquisadores, foi resolvida por meio de consenso. Realizou-se busca manual em outras fontes por artigos potencialmente elegíveis que porventura possam não ter sido identificados pelas estratégias de busca.

Os artigos passaram por leitura exploratória e os dados foram extraídos por meio de um formulário padronizado elaborada conforme recomendações da literatura ${ }^{(18)}$, considerando-se as variáveis de importância para a análise e aplicabilidade dos resultados.

Os ECR foram ainda submetidos à análise de qualidade metodológica. Dois revisores aplicaram o Escore Jadad ${ }^{(19)}$ visando à análise de qualidade e não exclusão da amostra final. Trata-se de um escore composto por cinco questões referentes a viés de pesquisa, como randomização, cegamento e destino de todos os participantes. Pontua-se valor zero quando o item não for atendido e um (01) quando o critério metodológico é atendido, quando somatório menor que três (03), indica-se que o ECR apresenta baixa qualidade metodológica e seus dados devem ser usados de forma cautelosa.

Os dados coletados foram organizados em quadros sínteses e passaram por análise de concordância e discordância de seu conteúdo, a fim de se identificarem as unidades de evidências a seres discutidas. A apresentação e discussão dos dados foi feita por meio de síntese qualitativa. 


\section{RESULTADOS}

A busca inicial resultou em 864 estudos na base de dados LILACS, 70 na CINAHL, 98 na SciELO, 1.147 na Web of Science, 4.172 na Scopus, 5.600 na Pubmed e 331 na BDENF, totalizando 12.492 publicações. A busca manual na lista de referências dos ECR selecionados e em veículos de busca online resultou em dois ECR potencialmente elegíveis para a análise. A seguir executou-se a seleção, considerando-se os relatos potencialmente elegíveis, nesta etapa 685 publicações foram eleitas. Desse total, 628 publicações foram excluídas por não se tratar de ECR. Restaram, então, 57 ECR para leitura na íntegra, contudo, pela leitura dos títulos e resumos, foram excluídos 38 estudos por não responderem à questão de pesquisa, dois com amostra de mulheres multíparas e oito duplicados, selecionando-se nove estudos ao final, os quais foram incluídos na amostra desta revisão, conforme ilustra a Figura 1.

Figura 1 - Fluxograma de seleção dos artigos incluídos. Timon, MA, Brasil, 2018

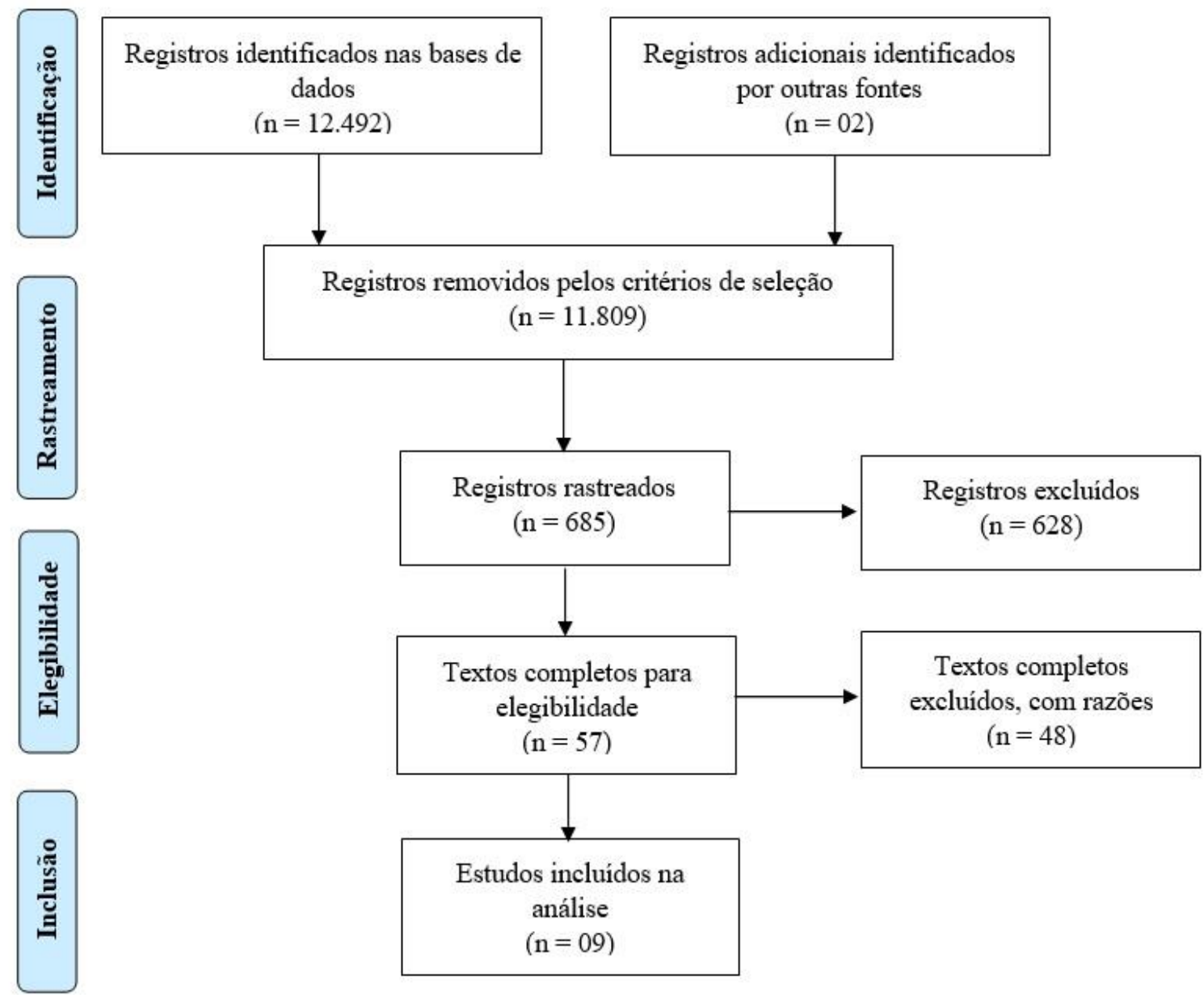


Fonte: Os autores

A análise dos nove artigos que compuseram a amostra evidenciou que a maior parte foi oriunda de periódicos internacionais e publicados na língua inglesa (oito). Cinco dos nove estudos foram considerados de boa qualidade (Escore Jadad $\geq 03$ ). Dois estudos utilizaram intervenção combinada, enquanto, os demais, intervenções isoladas. Da amostra total de nove artigos que foram incluídos, um obteve significância estatística para os dois períodos do tempo de TP de forma isolada, dois para a duração total do TP e parto, um para o período expulsivo de forma isolada e duração total do TP e parto e dois somente para o período expulsivo, os demais não obtiveram eficácia significativa (Quadro 1).

Quadro 1 - Estudos selecionados para o estudo. Timon, MA, Brasil, 2018

\begin{tabular}{|c|c|c|c|c|c|}
\hline $\begin{array}{c}\text { Título } \\
\text { Referência(*) }\end{array}$ & $\begin{array}{l}\text { Ano } \\
\text { País }\end{array}$ & $\begin{array}{l}\text { Delineamento } \\
\text { Número de } \\
\text { pacientes }\end{array}$ & Intervenções & Desfechos & $\begin{array}{l}\text { Escore } \\
\text { Jadad }\end{array}$ \\
\hline $\begin{array}{l}\text { A bola suíça no } \\
\text { alivio da dor de } \\
\text { primigestas na fase } \\
\text { ativa do trabalho } \\
\text { de parto } \\
\text { (20) }\end{array}$ & $\begin{array}{c}2014 \\
\text { Brasil }\end{array}$ & $\begin{array}{l}\text { ECR } \\
\mathrm{n}=40\end{array}$ & $\begin{array}{c}\text { Bola de Parto } \\
\text { versus } \\
\text { Cuidados } \\
\text { Habituais }\end{array}$ & $\begin{array}{l}\text { - Duração total do TP } \\
\text { e parto } \\
\text { Experimental }=2,75 \mathrm{~h} \\
\text { Controle }=3,0 \mathrm{~h}\end{array}$ & 01 \\
\hline $\begin{array}{l}\text { Effects of SP6 } \\
\text { acupuncture point } \\
\text { stimulation on } \\
\text { labor pain and } \\
\text { duration of } \\
\text { labor }^{(21)}\end{array}$ & $\begin{array}{c}2014 \\
\text { Turquia }\end{array}$ & $\begin{array}{c}\text { ECR } \\
n=100\end{array}$ & $\begin{array}{l}\text { Acupressão } \\
\text { versus } \\
\text { Cuidados } \\
\text { Habituais }\end{array}$ & $\begin{array}{c}\text { - Tempo de Dilatação } \\
\text { Intervenção=225 } \\
\text { minutos } \\
\text { Controle }=320 \text { minutos } \\
\text { - Tempo de Expulsão } \\
\text { Intervenção=15 } \\
\text { minutos } \\
\text { Controle }=20 \text { minutos }\end{array}$ & 03 \\
\hline $\begin{array}{l}\text { Effect of dance } \\
\text { labor on the } \\
\text { management of } \\
\text { active phase labor } \\
\text { pain and clients' } \\
\text { satisfaction: a } \\
\text { randomized } \\
\text { controlled trial } \\
\text { study }^{(2)}\end{array}$ & $\begin{array}{l}2014 \\
\text { Irã }\end{array}$ & $\begin{array}{l}\text { ECR } \\
n=60\end{array}$ & $\begin{array}{c}\text { Dança versus } \\
\text { Cuidados } \\
\text { Habituais }\end{array}$ & $\begin{array}{c}\text { Não informado dados } \\
\text { para o desfecho } \\
\text { quanto ao tempo }\end{array}$ & 02 \\
\hline $\begin{array}{l}\text { Comparison of the } \\
\text { effects of water } \\
\text { and traditional } \\
\text { delivery on } \\
\text { birthing women } \\
\text { and newborns }{ }^{(23)} \\
\end{array}$ & $\begin{array}{l}2015 \\
\text { China }\end{array}$ & $\begin{array}{c}\text { ECR } \\
n=120\end{array}$ & $\begin{array}{l}\text { Imersão em } \\
\text { Água versus } \\
\text { Cuidados } \\
\text { Habituais }\end{array}$ & $\begin{array}{l}\text { - Duração total do TP } \\
\text { e parto } \\
\text { Experimental }=5,27 \mathrm{~h} \\
\text { Controle }=6,11 \mathrm{~h}\end{array}$ & 01 \\
\hline
\end{tabular}




\begin{tabular}{|c|c|c|c|c|c|}
\hline $\begin{array}{l}\text { The effect of foot } \\
\text { reflexology on } \\
\text { anxiety, pain, and } \\
\text { outcomes of the } \\
\text { labor in } \\
\text { primigravida } \\
\text { women }^{(24)}\end{array}$ & $\begin{array}{c}2015 \\
\text { Irã }\end{array}$ & $\begin{array}{l}\text { ECR } \\
n=80\end{array}$ & $\begin{array}{l}\text { Reflexologia } \\
\text { Podal versus } \\
\text { Cuidados } \\
\text { Habituais }\end{array}$ & $\begin{array}{c}\text { Não informado dados } \\
\text { para o desfecho } \\
\text { quanto ao tempo }\end{array}$ & 01 \\
\hline $\begin{array}{l}\text { Effects of LI-4 and } \\
\text { SP-6 acupuncture } \\
\text { on labor pain, } \\
\text { cortisol level and } \\
\text { duration of } \\
\text { labor }^{(25)}\end{array}$ & $\begin{array}{l}2015 \\
\text { Irã }\end{array}$ & $\begin{array}{l}\text { ECR } \\
n=63\end{array}$ & $\begin{array}{c}\text { Acupuntura } \\
\text { profunda nos } \\
\text { pontos LI-4 e } \\
\text { SP-6 versus } \\
\text { Acupuntura } \\
\text { superficial } \\
\text { nos mesmos } \\
\text { pontos }\end{array}$ & $\begin{array}{c}\text { - Duração total do TP } \\
\text { e parto } \\
\text { Intervenção }=162 \\
\text { minutos } \\
\text { Controle }=280 \text { minutos } \\
\text { - Tempo de Expulsão } \\
\text { Intervenção }=130 \\
\text { minutos } \\
\text { Controle }=250 \text { minutos }\end{array}$ & 03 \\
\hline $\begin{array}{l}\text { Complementary } \\
\text { therapies for } \\
\text { labour and birth } \\
\text { study: a } \\
\text { randomised } \\
\text { controlled trial of } \\
\text { antenatal } \\
\text { integrative } \\
\text { medicine for pain } \\
\text { management in } \\
\text { labour }\end{array}$ & $\begin{array}{c}2016 \\
\text { Australia }\end{array}$ & $\begin{array}{c}\text { ECR } \\
n=171\end{array}$ & $\begin{array}{c}\text { Programa de } \\
\text { Educação } \\
\text { Pré-Natal } \\
\text { versus } \\
\text { Educação } \\
\text { Pré-Natal } \\
\text { rotineira }\end{array}$ & $\begin{array}{c}\text { - Tempo de Expulsão } \\
\text { Intervenção=1h } \\
\text { Controle }=1,32 \mathrm{~h}\end{array}$ & 05 \\
\hline $\begin{array}{l}\text { Birth ball or heat } \\
\text { therapy? A } \\
\text { randomized } \\
\text { controlled trial to } \\
\text { compare the } \\
\text { effectiveness of } \\
\text { birth ball usage } \\
\text { with sacrum } \\
\text { perineal heat } \\
\text { therapy in labor } \\
\text { pain } \\
\text { management }\end{array}$ & $\begin{array}{l}2016 \\
\text { Irã }\end{array}$ & $\begin{array}{l}\text { ECR } \\
n=87\end{array}$ & $\begin{array}{l}\text { 01: Terapia } \\
\text { de Calor } \\
\text { (compressas } \\
\text { e toalhas) } \\
\text { versus } \\
\text { Cuidados } \\
\text { Habituais } \\
\text { 02: Bola de } \\
\text { Parto versus } \\
\text { Cuidados } \\
\text { Habituais }\end{array}$ & $\begin{array}{c}\text { - Tempo de Dilatação } \\
\text { Intervenção } 01=1,95 \mathrm{~h} \\
\text { Intervenção } 02=1,78 \mathrm{~h} \\
\text { Controle }=1,67 \mathrm{~h}\end{array}$ & 04 \\
\hline $\begin{array}{l}\text { Sequential } \\
\text { application of non } \\
\text { pharmacological } \\
\text { interventions } \\
\text { reduces the } \\
\text { severity of labour } \\
\text { pain, delays use of } \\
\text { pharmacological } \\
\text { analgesia, and } \\
\text { improves some }\end{array}$ & $\begin{array}{c}2018 \\
\text { Brasil }\end{array}$ & $\begin{array}{l}\text { ECR } \\
n=80\end{array}$ & $\begin{array}{c}\text { Bola de Parto } \\
\text { + Massagem } \\
\text { Lombossacral } \\
\text { + Banho de } \\
\text { Aspersão } \\
\text { versus } \\
\text { Cuidados } \\
\text { Habituais }\end{array}$ & $\begin{array}{c}\text { - Tempo de Expulsão } \\
\text { Intervenção=19 } \\
\text { minutos } \\
\text { Controle }=37 \text { minutos }\end{array}$ & 05 \\
\hline
\end{tabular}


obstetric

outcomes: a

randomized trial $^{(28)}$

Nota: ECR: Ensaio clínico randomizado. TP: Trabalho de parto.

\section{DISCUSSÃO}

Esta pesquisa, ao avaliar a eficácia das TNF sobre a redução do tempo de TP e parto, evidenciou que acupressão, reflexologia podal e banho de imersão em água possuem potencial efetivo para a redução do tempo total de TP e parto, enquanto programas de educação pré-natal e intervenção combinada com uso de bola de parto, massagens e banho de aspersão em água a $37^{\circ} \mathrm{C}$ para o tempo de

Dentro as tecnologias aplicadas, a acupressão mostrou resultados significativos para a duração total do TP e parto com uma redução média de 100 minutos quando comparado ao grupo controle ${ }^{(21)}$. Não são claros os mecanismos de ação que provocam tais achados, contudo, sabe-se que o ponto BP6 possui forte influência sobre os órgãos reprodutivos, sendo indicado para estimulação em TP distócicos e prolongados ${ }^{(15)}$.

Durante o TP os meridianos, sistemas de canais no corpo por onde flui a energia vital e regulação dos sistemas orgânicos, possuem seu fluxo reduzido provocando malestar nas parturientes, acredita-se que a acupressão no ponto BP-6 provoque desobstrução do fluxo ao tempo que promove bem-estar, ainda que tal estímulo aumente a período expulsivo, e a acupuntura tanto para a duração total do TP e parto quanto ao segundo estágio do TP.

Observou-se que as TNF são utilizadas como métodos auxiliares na assistência ao TP e parto, sendo que os estudos, em sua maioria, as descrevem de maneira isolada ou em comparação às outras tecnologias, analisandose principalmente sua eficácia para o alívio da dor no TP.

liberação de ocitocina pela glândula pituitária, melhorando a evolução do TP por meio da regulação das contrações uterinas ${ }^{(29)}$.

Assim como para a acupressão, os mecanismos elucidativos do efeito sobre o tempo de TP e parto para a acupuntura não estão claros. Estudos demonstraram eficácia da acupuntura sobre o tempo de $\mathrm{TP}^{(25-26)}$. Para clarear tal efeito estudo aponta que a acupuntura aumenta a liberação de ocitocina pela glândula pituitária anterior, além de reduzir a necessidade de ocitocina para induzir o $\mathrm{TP}^{(30)}$.

Sugere-se, desta forma, outras investigações a fim de se elucidar tais mecanismos, tendo em vista não se identificar estudos de mensuração dos níveis de ocitocina quando da estimulação do ponto 
BP-6 tanto por acupressão quanto por acupuntura.

Quanto à reflexologia podal, os achados podem ser explicados pelo efeito contrário ao do relaxamento em virtude da diminuição das concentrações de adrenalina e noradrenalina provocado por essa TNF, aumentando os níveis de endorfinas e ocitocina, podendo afetar a duração do TP e parto pelos efeitos da ocitocina sobre as contrações uterinas e atividade muscular uterina ${ }^{(31)}$. Achados semelhantes foram identificados em outro estudo iraniano ${ }^{(32)}$.

O estudo que propôs analisar o banho de imersão durante o TP evidenciou que a duração total do TP e parto obteve uma diferença de 50,4 minutos inferior ao TP tradicional a seco ${ }^{(23)}$. Esta prática só deve ser recomendada quando o TP está instalado evitando-se desaceleração do TP pelo efeito relaxante provocado pela água. Só podem receber, essa tecnologia, parturientes a partir da $38^{\mathrm{a}}$ semana de gestação, sem anormalidades ou complicações obstétricas, batimentos cardiofetais normais $\mathrm{e}$ apresentação cefálica, sendo contraindicada para mulheres com gravidez gemelar, mau posicionamento fetal, macrossomia fetal e líquido amniótico contaminado.

Uma das contribuições das pesquisas revisadas foi $\mathrm{o}$ enfoque à educação permanente durante o pré-natal. Programas de educação em saúde mostram-se importantes aliados durante o TP, pois, promovem relaxamento e fortalecimento da autonomia da mulher, reduzindo os medos e angústias quanto ao processo parturitivo.

Estudo mediante práticas de relaxamento com posturas de Yoga, exercícios respiratórios, massagem e apoio do parceiro demonstrou redução significativa no tempo do período expulsivo e aumento da satisfação da mulher com a experiência do nascimento $^{(26)}$. Outro estudo com uso de intervenções combinadas, também, demonstrou resultado significativo para o período expulsivo do $\mathrm{TP}^{(28)}$.

Entre as intervenções utilizadas de forma combinada estavam: exercícios respiratórios, bola de parto, banho de aspersão em água a $37^{\circ} \mathrm{C}$ e massagens.

Pesquisa transversal com 188 primíparas identificou como a TNF mais utilizada o suporte emocional do acompanhante, seguido por banhos de aspersão, exercícios respiratórios, mudanças de posição, bola de parto, massagens manuais, musicoterapia, massagens com equipamentos e sentar em cadeira ${ }^{(33)}$. Outro estudo identificou deambulação e bola suíça (bola de parto) como as tecnologias mais utilizadas por um grupo de primíparas no Rio Grande do $\mathrm{Sul}^{(1)}$.

Os benefícios dos exercícios respiratórios lentos e profundos do tipo diafragmático e das massagens na região 
lombossacral se dão em forma de relaxamento muscular, sensação de bem-estar físico e emocional, proporcionando tranquilidade e maior interação com a equipe durante o $\mathrm{TP}^{(29)}$.

Em relação à bola de parto, os benefícios decorrem da adoção da posição verticalizada, que promove maior alinhamento do eixo fetal com a pelve materna, favorece a força da gravidade e auxilia na progressão da descida fetal através do canal de parto por meio do relaxamento muscular devido aos exercícios perineais, além de melhorar a circulação uterina aumentando a intensidade das contrações ${ }^{(14)}$.

Os banhos quentes, sejam de imersão ou aspersão, apontam como uma das TNF mais utilizada durante o processo parturitivo. Os benefícios estão na promoção de relaxamento, redução dos medos, ansiedade, tensão e estresse, alívio da dor e inibição do sistema nervoso simpático, com consequente redução dos níveis de catecolaminas ${ }^{(34)}$. Estudo apontou aumento significativo da dilatação entre parturientes que utilizaram do banho quente como TNF para alívio da dor, sugerindo abreviação do tempo de TP e parto $^{(35)}$.

Algumas limitações desta revisão devem ser consideradas: o número reduzido de ECR para análise, o uso do filtro por idiomas, o balizamento temporal e a diversidade clínica das intervenções que dificultou a comparação entre resultados e execução de metanálise.

As dificuldades de comparação entre os resultados, em parte, ocorreram em vista à diversidade metodológica e clínica dos ECR analisados. Contudo, no que diz respeito à qualidade metodológica, observou-se que a maioria apresentou boa qualidade, o que reforça as evidências aqui apresentadas.

Ao mesmo tempo, reforça-se a necessidade de padronização de instrumentos para mensuração dos desfechos, o que facilitaria as sumarizações e estudos futuros sobre a temática, tendo em vista que a escolha do instrumento de mensuração deve obedecer a critérios rigorosos de elegibilidade e compreensão da população a ser investigada.

De modo mais aprofundado, um estudo utilizou o nível de cortisol como indicador de intensidade da dor durante o trabalho de parto, minimizando assim as limitações postas pelo uso das escalas visuais e analógicas da dor, instrumento utilizado na maioria dos estudos, e de cunho subjetivo ${ }^{(25)}$.

Além disso, identificou-se que algumas informações necessárias para considerar ou não válidas as evidências para a aplicação na prática foram suprimidas, em alguns estudos, como o processo de randomização, o mascaramento, os instrumentos usados para medir os desfechos e a amplitude do efeito das intervenções, prendendo-se, em parte, somente ao "estatisticamente significativo". 
Não obstante, deve-se considerar que resultados não significativos possam ser importantes do ponto de vista clínico, e ainda serem decorrentes de vieses metodológicos, como número reduzido de participantes, perdas de seguimento, instrumentos de mensuração e alocação dos participantes, portanto, deve-se evitar a ocultação de informações nos relatórios de pesquisa.

Ainda, apresenta limites inerentes à estratégia de busca que poderia ter sido ampliada com a inclusão de termos que identificassem as intervenções e os desfechos analisados nos ECR, como, por exemplo, em estudo de revisão ${ }^{(36)}$ que identificou o apoio durante o nascimento como tecnologia que reduz a duração do $\mathrm{TP}$, o número de cesarianas, uso de qualquer analgesia e sentimentos negativos de experiência durante o parto, ratificando a necessidade de ampliação de termos de busca em investigações futuras.

Apesar das limitações apresentadas, esse estudo fomenta as discussões acerca da implementação das boas práticas de atenção ao parto e nascimento, recomendadas internacionalmente e inseridas nas políticas públicas de saúde do Brasil, contribuindo assim, também, para a enfermagem como categoria profissional inserida neste cenário.

Os resultados, ainda, mostram-se relevantes ao passo que apontam caminhos para uma assistência humanizada ao processo parturitivo, incentivando uma prática clínica baseada em evidências científicas, com o uso das TNF de alívio da dor durante o TP e parto abreviando sua duração e promovendo a integralidade do cuidado. Aponta, também, lacunas na produção de conhecimento a serem investigadas futuramente, de modo a buscar o aprimoramento da assistência à mulher em TP e parto.

\section{CONCLUSÃO}

Com base nos achados dessas pesquisas observa-se que os exercícios respiratórios, massagem lombossacral, acupuntura, acupressão, banho quente $\left(37^{\circ} \mathrm{C}\right)$, exercícios perineais em bola de parto, programas de educação no pré-natal e reflexologia podal, aplicadas de forma isolada ou combinada, são tecnologias em potencial para o alívio da dor de parturientes e redução significativa do tempo de TP e parto.

Apesar das limitações e dos resultados inconclusivos face às deficiências metodológicas, a presente revisão demonstrou que as TNF podem ser eficazes na redução do tempo de TP e parto; por conseguinte, tornase relevante a realização de outros estudos que analisem o impacto das TNF no tempo de TP e parto, sobretudo utilizando-se de metodologia padronizada para confirmar estes achados, bem como o uso de indicadores específicos para os desfechos a serem analisados. 


\section{REFERÊNCIAS}

1. Scarton J, Ressel LB, Siqueira $\mathrm{HCH}$, Rangel RF, Tolfo F, Weykamp JM. Care practices in normal birth: the experience of primiparous women. J Res Fundam Care Online [Internet]. 2018 [acesso em 16 jan 2019]; 10(1):17-24. Disponível em: http://dx.doi.org/10.9789/21755361.2018.v10i1.17-24

2. World Health Organization. Appropriate Technology for Birth. Lancet [Internet]. 1985 [acesso em 16 jan 2019]; 2(8452):436-7. Disponível: http://dx.doi.org/10.1016/S01406736(85)92750-3

3. World Health Organization. Care in normal birth: a practical guide. Birth [Internet]. 1997 [acesso em 16 jan 2019]; 24(2):121-3. Disponível

em: https://www.ncbi.nlm.nih.gov/pubmed/92719 $\underline{79}$

4. Brasil. Ministério da Saúde. Diretrizes Nacionais de Assistência ao Parto Normal: versão resumida [Internet]. Brasília; 2017 [acesso em 16 jan 2019]. Disponível em: http://bvsms.saude.gov.br/bvs/publicacoes/dir etrizes_nacionais_assistencia_parto_normal.p df

5. Leal MC, Pereira APE, Domingues RMSM, Theme Filha MM, Dias MAB, Nakamura-Pereira $M$, et al. Obstetric interventions during labor and childbirth in Brazilian low-risk women. Cad Saúde Pública [Internet]. 2014 [acesso em 16 jan 2019];
30(supp11):S17-S32. Disponível em:

http://dx.doi.org/10.1590/0102-

$\underline{311 X 00151513}$

6. Cortês CT, Oliveira SMJV, Santos RCS, Francisco AA, Riesco MLG, Shimoda GT. Implementation of evidence-based practices in normal delivery care. Rev Latino-Am Enfermagem [Internet]. 2018 [acesso em 16 jan 2019]; 26:e2988. Disponível em: http://dx.doi.org/10.1590/1518-

\section{$\underline{8345.2177 .2988}$}

7. Sandall J, Soltani H, Gates S, Shennan A, Devane D. Midwife-led continuity models versus other models of care for childbearing women. Cochrane Database Syst Rev [Internet]. 2015 [acesso em 16 jan 2019]; 9:[10 telas]. Disponível em: http://cochrane.bvsalud.org/doc.php?db=revie $\underline{\text { ws\&id=CD004667 }}$

8. Sousa AMM, Souza KV, Rezende EM, Martins EF, Campos D, Lansky S. Practices in childbirth care in maternity with inclusion of obstetric nurses in Belo Horizonte, Minas Gerais. Esc Anna Nery [Internet]. 2016 [acesso em 16 jan 2019]; 20(2):324-31. Disponível em: http://dx.doi.org/10.5935/1414-

$\underline{8145.20160044}$

9. Scarton J, Prates LA, Wilhelm LA, Silva SC, Possati AB, Ilha CB, et al. "It was worth it when I saw his face": experiences of primiparous women during natural childbirth. Rev Gaúcha Enferm [Internet]. 2015 [acesso 
em 16 jan 2019]; 36(esp):143-51. Disponível em: $\quad$ http://dx.doi.org/10.1590/1983$\underline{1447.2015 . e s p .56786}$

10. Kottwitz F, Gouveia HG, Gonçalves AC. Route of birth delivery preferred by mothers and their motivations. Esc Anna Nery [Internet]. 2018 [acesso em 16 jan 2019]; 22(1):e20170013. Disponível em: http://dx.doi.org/10.1590/2177-9465-EAN$\underline{2017-0013}$

11. Osório SMB, Silva Junior LG, Nicolau AIO. Assessment of the effectiveness of nonpharmacological methods in pain relief during labor. Rev Rene [Internet]. 2014 [acesso em 16 jan 2019]; 15(1):174-84. Disponível em: http://dx.doi.org/10.15253/2175-

\section{$\underline{6783.2014000100022}$}

12. Mafetoni RR, Shimo AKK. Nonpharmacological methods for pain relief during labor: integrative review. REME Rev Min Enferm [Internet]. 2014 [acesso em 16 jan 2019]; 18(2):505-12. Disponível em: http://dx.doi.org/10.5935/1415-

\subsection{7}

13. Melo LPT, Pereira AMM, Rodrigues DP, Dantas SLC, Ferreira ALA, Fontenele FMC, et al. Representações de puérperas sobre o cuidado recebido no trabalho de parto e parto. Av Enferm [Internet]. 2018 [acesso em 16 jan 2019]; 36(1):22-30. Disponível em: http://dx.doi.org/10.15446/av.enferm.v36n1.6 $\underline{3993}$
14. Henrique AJ, Gabrielloni MC, Cavalcanti ACV, Melo PS, Barbieri M. Hydrotherapy and the Swiss ball in labor: randomized clinical trial. Acta Paul Enferm [Internet]. 2016 [acesso em 16 jan 2019]; 29(6):686-92. Disponível em:

\section{http://dx.doi.org/10.1590/1982-}

\section{$\underline{0194201600096}$}

15. Mafetoni RR, Shimo AKK. The effects of acupressure on labor pains during child birth: randomized clinical trial. Rev Latino-Am Enfermagem [Internet]. 2016 [acesso em 16 jan 2019]; 24:e2738. Disponível em: http://dx.doi.org/10.1590/1518-

\section{$\underline{8345.0739 .2738}$}

16. Dantas SLC, Oliveira GYM, Costa KFL, Barros AA, Chaves EMC, Carvalho REFL. Experimental studies in the gestational period: an overview of scientific production. Rev Esc Enferm USP [Internet]. 2018 [acesso em 16 jan 2019]; 52:e03325. Disponível em: http://dx.doi.org/10.1590/S1980-

\section{X2017024403325}

17. Galvão TF, Pansani TSA, Harrad D. Principais itens para relatar Revisões Sistemáticas e Meta-análises: a recomendação PRISMA. Epidemiol Serv Saúde [Internet]. 2015 [acesso em 16 jan 2019]; 24(2):335-42. Disponível em: http://scielo.iec.gov.br/scielo.php?script=sci arttext\&pid=S1679$\underline{49742015000200017 \& \operatorname{lng}=\text { pt\&nrm=iso\&tlng }}$ $=\mathrm{pt}$ 
18. Brasil. Ministério da Saúde. Diretrizes metodológicas: elaboração de revisão sistemática e metanálise de ensaios clínicos randomizados [Internet]. Brasília; 2015 [acesso em 16 jan 2019]. Disponível em: http://bvsms.saude.gov.br/bvs/publicacoes/dir etrizes_metodologicas_ensaio_clinico_rando mizado_1edicao.pdf

19. Jadad AR, Moore RA, Carroll D, Jenkinson C, Reynolds DJ, Gavaghan DJ, et al. Assessing the quality of reports of randomized clinical trials: is blinding necessary? Control Clin Trials [Internet]. 1996 [acesso em 16 jan 2019]; 17(1):1-12. Disponível

em:

http://www.sciencedirect.com/science/article/ pii/0197245695001344

20. Gallo RBS, Santana LS, Marcolin AC, Quintana SM. Swiss ball to relieve pain of primiparous in active labor. Rev Dor [Internet]. 2014 [acesso em 16 jan 2019]; 15(4):253-5. Disponível em: http://dx.doi.org/10.5935/1806-

\section{$\underline{0013.20140054}$}

21. Calik KY, Komurcu N. Effects of SP6 acupuncture point stimulation on labor pain and duration of labor. Iran Red Crescent Med J [Internet]. 2014 [acesso em 16 jan 2019]; 16(10):e16461. Disponível em: http://dx.doi.org/10.5812/ircmj.16461

22. Abdolahian S, Chavi F, Abdollahifard S, Sheikhan F. Effect of dance labor on the management of active phase labor pain \& clients' satisfaction: a randomized controlled trial study. Global Journal of Health Science [Internet]. 2014 [acesso em 16 jan 2019]; 6(3):219-26. Disponível em: http://dx.doi.org/10.5539/gjhs.v6n3p219

23. Gayiti MRY, Li XY, Zulifeya AK, Huan Y, Zhao TN. Comparison of the effects of water and traditional delivery on birthing women and newborns. Eur Rev Med Pharmacol Sci [Internet]. 2015 [acesso em 16 jan 2019]; 19(9):1554-8. Disponível em: https://www.europeanreview.org/article/8873

24. Hanjani SM, Tourzani ZM, Shaghi M. The effect of foot reflexology on anxiety, pain, and outcomes of the labor in primigravida women. Acta Medica Iranica [Internet]. 2015 [acesso em 16 jan 2019]; 53(8):507-11. Disponível em: http://acta.tums.ac.ir/index.php/acta/article/vie w/4277

25. Asadi N, Maharlouei N, Khalili A, Darabi Y, Davoodi S, Shahraki HR, et al. Effects of LI-4 and SP-6 acupuncture on labor pain, cortisol level and duration of labor. $\mathbf{J}$ Acupunct Meridian Stud [Internet]. 2015 [acesso em 16 jan 2019]; 8(5):249-54. Disponível

em: http://dx.doi.org/10.1016/j.jams.2015.08.003 26. Levett KM, Smith CA, Bensoussan A, Dahlen HG. Complementary therapies for labour and birth study: a randomised controlled trial of antenatal integrative medicine for pain management in labour. 
BMJ Open [Internet]. 2016 [acesso em 16 jan 2019]; 6:e010691. Disponível em: http://dx.doi.org/10.1136/bmjopen-2015-

$\underline{010691}$

27. Taavoni S, Sheikhan F, Abdolahian S, Ghavi F. Birth ball or heat therapy? A randomized controlled trial to compare the effectiveness of birth ball usage with sacrum perineal heat therapy in labor pain management. Complementary Therapies in Clinical Practice [Internet]. 2016 [acesso em 16 jan 2019]; 24: 99-102. Disponível em: http://dx.doi.org/10.1016/j.ctcp.2016.04.001

28. Gallo RBS, Santana LS, Marcolin AC, Duarte G, Quintana SM. Sequential application of non-pharmacological interventions reduces the severity of labour pain, delays use of pharmacological analgesia, and improves some obstetric outcomes: a randomised trial. Journal of Physiotherapy [Internet]. 2018 [acesso em 16 jan 2019]; 64:33-40. Disponível em: http://dx.doi.org/10.1016/j.jphys.2017.11.014 29. Mafetoni RR, Shimo AKK. Effects of acupressure on progress of labor and cesarean section rate: randomized clinical trial. Rev Saúde Pública [Internet]. 2015 [acesso em 16 jan 2019]; 49:[09 telas]. Disponível em: http://dx.doi.org/10.1590/S0034$\underline{8910.2015049005407}$

30. Gaudernack LC, Forbord S, Hole E. Acupuncture administered after spontaneous rupture of membranes at term significantly reduces the length of birth and use of oxytocin: a randomized controlled trial. Acta Obstet Gynecol Scand [Internet]. 2006 [acesso em 16 jan 2019]; 85(11):1348-53. Disponível

em: http://dx.doi.org/10.1080/0001634060093583 $\underline{9}$

31. Alehagen S, Wijma B, Lundberg U, Wijma K. Fear, pain and stress hormones during childbirth. J Psychosom Obstet Gynaecol [Internet]. 2005 [acesso em 16 jan 2019]; 26(3):153-65. Disponível em: http://dx.doi.org/10.1080/0144361040002307 $\underline{2}$

32. Dolatian M, Hasanpour A, Montazeri S, Heshmat R, Majd HA. The effect of reflexology on pain intensity and duration of labor on primiparas. Iran Red Crescent Med J [Internet]. 2011 [acesso em 16 jan 2019]; 13(7):475-9. Disponível em: https://www.ncbi.nlm.nih.gov/pmc/articles/P $\underline{\text { MC3371987/ }}$

33. Gayeski ME, Brüggemann OM, Monticelli M, Santos EKA. Application of nonpharmacologic methods to relieve pain during labor: the point of view of primiparous women. Pain Management Nursing [Internet]. 2015 [acesso em 16 jan 2019]; 16(3):273-84. Disponível em: http://dx.doi.org/10.1016/j.pmn.2014.08.006 34. Barbieri M, Henrique AJ, Chors FM, Maia NL, Gabrielloni MC. Warm shower aspersion, perineal exercises with Swiss ball 
and pain in labor. Acta Paul Enferm [Internet]. 2013 [acesso em 16 jan 2019]; 26(5):478-84. Disponível em: http://dx.doi.org/10.1590/S0103-

21002013000500012

35. Lee SL, Liu CY, Lu YY, Gau ML. Efficacy of warm showers on labor pain and birth experiences during the first labor stage. $\mathrm{J}$ Obstet Gynecol Neonatal Nurs [Internet]. 2013 [acesso em 16 jan 2019]; 42(1):19-28.

Disponível em: http://dx.doi.org/10.1111/j.1552$\underline{6909.2012 .01424 . x}$
36. Bohren, MA, Hofmeyr GJ, Sakala C, Fukuzawa RK, Cuthbert A. Continuous support for women during childbirth. Cochrane Database Syst Rev [Internet]. 2017 [acesso em 16 jan 2019]; (7):CD003766. Disponível

em: http://dx.doi.org/10.1002/14651858.CD00376 6.pub6

Submissão: 2020-06-29

Aprovado: 2021-01-06 